\title{
Bedenkliche Standespolitik
}

\section{Daniel Bracher}

Dr. med., Mitglied FMH

\author{
Mit dem Ruf nach Kostenneutralität lösten Krankenkassen und Politiker einen \\ Kampf zwischen den Fachgesellschaften aus und verringerten das Entgelt für ärzt- \\ liche Leistungen. Eindeutig besser wäre es, wenn die Standespolitik erfassen würde, \\ bei welchen Krankheiten sich die Kosten wegen des medizinischen Fortschritts \\ oder der Überalterung erhöhen und wo sie sinken.
}

\section{Der TARMED ist ein standes- politisches Grab}

TARMED hat nebst jährlichen Kosten pro Praxis von ca. 1500 CHF zwei schwere Mängel: Zum einen ist die Annahme falsch, der Wert ärztlicher Leistungen lasse sich betriebswirtschaftlich berechnen. Wäre dies der Fall, so hätte die Erstellung des TARMED nicht fünfzehn Jahre (und die Revision nun vier Jahre) gedauert und trotzdem zu völlig schiefen Ergebnissen geführt. (Musterbeispiel: Die Gastroenterologen verdienen nahezu doppelt so viel wie die verwandte Gruppe der Endokrinologen.)

Zum anderen wurden nicht nur tausende Leistungen auf den Rappen genau falsch berechnet, sondern es bleibt offen, ob die erbrachten Leistungen nötig oder entbehrlich waren. Der Beitrag des TARMED zur Effizienzsteigerung ist null, und damit schufen sich die

\section{De la politique professionnelle}

Le TARMED creuse la tombe de la politique professionnelle. En exigeant la neutralité des coûts, les caisses maladie et les politiciens ont réussi à enflammer les relations entre les sociétés de discipline, à mettre le cap sur un budget global et à diminuer la rémunération des prestations médicales. Or il serait nettement préférable que la politique professionnelle se serve des trust center mis en place par le corps médical pour saisir à l'interne quels coûts de quelles maladies baissent ou augmentent avec les progrès de la médecine et quels traitements suivent la courbe du vieillissement de la population. En parallèle, le corps médical devrait enfin agir pour moins de traitements superflus en faisant notamment vérifier les indications des traitements diagnostiques ou thérapeutiques par les sociétés de discipline médicale. Ceci n'est pas une limitation de la liberté de traitement; car être libre signifie "décider en connaissance de cause», et chaque médecin doit être en mesure de rendre des comptes. "Faire et laisser faire» ne nourrit pas la liberté mais l'arbitraire. Un retour à la raison s'impose.
Ärzte politisch ein Grab; denn die Kassen riefen «Kostenneutralität» und konnten nun zuschauen, wie die FMH in Fachgesellschaften zerfiel und sich in hunderten von Arbeitsstunden stritt. Und die Politik folgte dem Ruf, war dem ambulanten Globalbudget einen wichtigen Schritt näher gekommen und setzte noch einen obendrauf, indem sie die Voraussetzung schuf, selber den TARMED zu revidieren und dazu das Bundesamt für Gesundheit um sechs (!) Vollzeitstellen aufstockte.

\section{Die Einsicht über das Wesen der Politik ...}

Politik ist das Bestreben, sich und seinesgleichen wirtschaftliche Vorteile zu verschaffen und zu erhalten. Bismarck wird die Aussage zugeschrieben: «Eine Revolution wird von den Philosophen vorbereitet, von Fanatikern durchgeführt, und die Halunken ernten die Früchte», aber das sind nur Spielarten; die von den Philosophen geforderten Prinzipien kommen ohne wirtschaftliche Freiheit nicht zum Tragen, und bei den Halunken ist ohnehin klar, worum es ihnen geht. Es gibt jedoch drei Variablen: Erstens, wie weit oder wie eng "seinesgleichen" gefasst wird. Zweitens, ob man das Wohlergehen anderer mitberücksichtigt bzw. wie rücksichtslos man seine Ansprüche durchzusetzen sucht. Und drittens, ob man seine wirtschaftlichen Ansprüche begründet oder tarnt, indem man der Bevölkerung anderes vorgaukelt.

\section{... übertragen auf die Standespolitik}

Mit einem Anteil von zwei Promille an der Gesamtbevölkerung kann die Ärzteschaft «seinesgleichen» nicht weit fassen, und wenn sich ein Promille Grundversorger mit einem Promille Spezialisten streiten, 


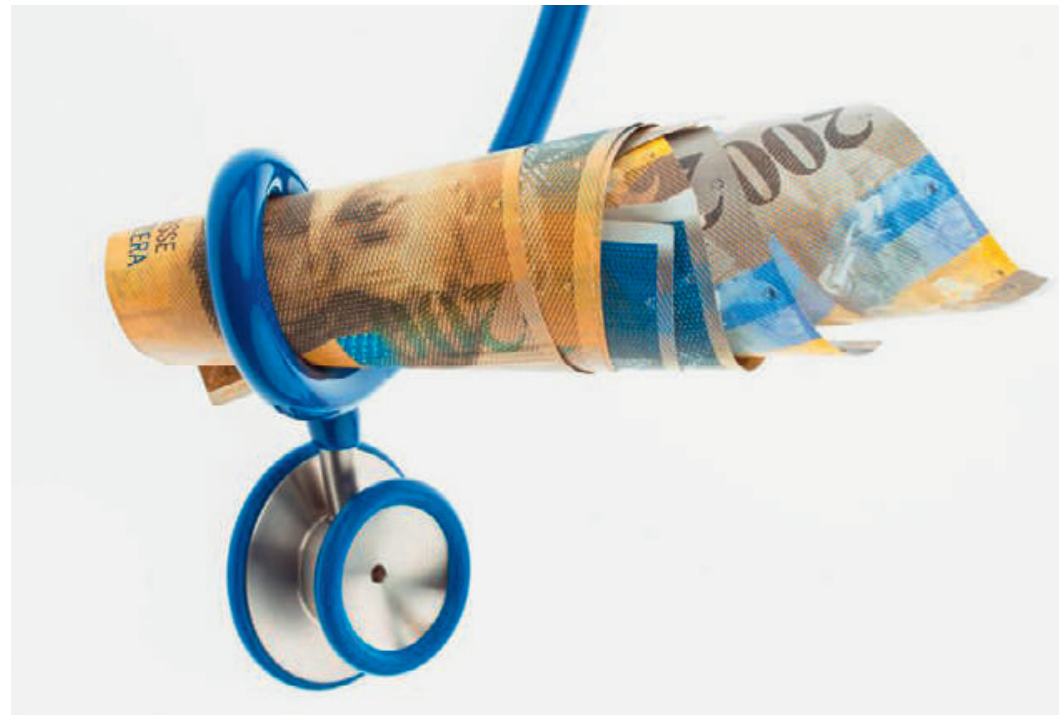

Es braucht ernsthafte Anstrengungen, um das Gesundheitswesen effizienter zu gestalten.

rückt der politische Erfolg in noch weitere Ferne. Folglich muss versucht werden, dem Wohlergehen der Bevölkerung sichtbar zu dienen, aber gute medizinische Leistung genügt nicht mehr; denn die Mediziner werden durch ihren Erfolg bestraft. Der Laie glaubt heute an ein Recht, gesund zu sein, und sollte er doch einmal erkranken, gilt «sano subito».

\section{Im Grunde ist allen Ärzten klar, dass sie aktiv werden müssen.}

Als Problem empfunden werden hingegen die hohen und übermässig steigenden Kosten für die Grundversicherung, und hier versagt die Standespolitik. Das Anliegen ist alt und eine Abstumpfung verständlich, wären da nicht die untrüglichen Zeichen einer Zuspitzung des Problems und seine Begleiterscheinungen.

\section{Zuspitzung:}

- Die Zahl der Versicherten, die sich eine Zusatzversicherung "privat» oder «halbprivat» leisten, nimmt kontinuierlich ab.

- Die 1996 in Kraft getretene Revision des KVG übertüncht nur die aus dem Ruder laufenden Kosten.

- Die Subventionen der Eidgenossenschaft an die Krankenkassen betrugen 1995 307,7 Millionen Franken, wurden ab 1996 in Prämienverbilligung umgewandelt und beliefen sich 2015 auf 2356 Milliarden Franken oder $+765,7 \%$ (und die Kantone zahlen nochmals über 2 Milliarden).

- Die Einheitsprämie ab dem Alter von 26 Jahren bedeutet, dass die meist gesunden werktätigen
Erwachsenen wesentlich mehr für die Senioren bezahlen, als diese zu ihrer Zeit an die damals Älteren bezahlt haben. Klugscheisser mögen das "dynamisch interpretierte Solidarität» nennen, treffender ist die Bezeichnung "GenerationenSchneeballsystem", und solche funktionieren langfristig nie.

\section{Begleiterscheinungen:}

Alle standespolitischen Probleme sind Folge der Betriebsamkeit von Politikern und Ökonomen, weil sich die Ärzte um die überproportional steigenden Gesundheitskosten foutieren. Alles, was die Ärzteschaft zu bieten hat, ist Desinteresse, Fatalismus, nur bedingt gültige Schutzbehauptungen («alles ist Folge der Überalterung») oder sogar dümmliche Ausflüchte, wie die Schweizer gäben mehr Geld für Ferien aus als für die Krankenkasse. Meine Damen und Herren: In einem mittleren Kanton wie Bern lag 2014 eine Familie mit zwei Kindern unter 16 Jahren bei einem steuerbaren Einkommen von 81000 CHF auf der 75. Perzentile, und mit 86000 CHF auf der 80. Sie bezahlte Steuern von 14500 CHF bzw. 16200 CHF und Prämien für die Grundversicherung der Krankenkasse von ca. $2 \times 350 \mathrm{CHF}$ plus $2 \times 100$ CHF monatlich, also ca. 10800 CHF im Jahr, und bei den heutigen Mietkosten etc. konnte sie nicht 11000 CHF für Ferien erübrigen, und die weniger verdienenden $75 \%$ bzw. $80 \%$ konnten es erst recht nicht.

\section{Die Bereitschaft zu sinnvollem Vorgehen}

Im Grunde ist allen Ärzten klar, dass sie aktiv werden müssen [1, 2]. Der FMH-Vorstand kann jedoch nur tun, was das Fussvolk will, und dieses muss aufhören, stets nur an das eigene Gärtlein zu denken.

Der einfachste Weg ist der, auf Entbehrliches zu verzichten, was aber nur geht, wenn der einzelne Arzt bereit ist, Rechenschaft für sein Tun abzulegen, und dagegen ist der Widerstand heftig, aber unbegründet; denn Freiheit heisst "mit Sachkenntnis entscheiden", und wer das tut, kann auch Rechenschaft ablegen, während «tun und lassen, was einem beliebt» Willkür ist. Auch das Arztgeheimnis bliebe gewahrt; denn die klügste standespolitische Errungenschaft der letzten Jahrzehnte ist die Schaffung der trust center, und diese können Angaben nur filtriert an die Versicherungen weiterleiten.

Man beachte auch: Der Bundesrat schätzt in Gesundheit2020 die möglichen Einsparungen durch Verzicht auf Entbehrliches auf 20\% der Gesundheitskosten. Rücken die Ärzte nicht selber dem Problem zu Leibe oder widerlegen es, so werden die üblichen Verdächtigen über kurz oder lang mit weniger tauglichen Mit- 
teln aktiv (Aufhebung des Kontrahierungszwangs usw.). Choosing Wisely ist ein richtiger Schritt, aber als blosser Aufruf zu zaghaft. Drei Schritte sind nötig.

\section{Beschreibung der Behandlung}

Auf jeder Honorarforderung ist anzugeben: 1) Was führte zur Behandlung? 2) Hatte die Patientin vorbestehende Leiden? und 3) Welche Arbeitshypothese wählte die Ärztin? Diese Angaben könnte die MPA in mindestens $80 \%$ der Fälle anhand eines Spickzettels aus zwei A4-Seiten einsetzen [2]. Die Richtigkeit der Angaben ist (z.B. durch ein Gremium von Kollegen, die eben in den Ruhestand gewechselt haben) zu überprüfen und auszuwerten und das Ergebnis wäre erhellend: Für Gesunde mit Kopfschmerzen, bei welchen sich die Vermutung eines Hirntumors bestätigt, werden sich bei allen Ärztinnen zwar hohe, aber ähnlich hohe Kosten (bzw. eine schlanke Gauss'sche Kurve) ergeben; dort hingegen, wo Spannungskopfschmerzen vermutet werden und es dabei bleibt, werden sehr unter-

\section{Choosing Wisely ist ein richtiger Schritt, aber als blosser Aufruf zu zaghaft.}

schiedliche Kosten (und wenn überhaupt eine Gauss'sche Kurve, so eine breite, flache) resultieren. Je breiter und flacher die Kurve ist, desto wahrscheinlicher wird von denjenigen in der rechten Kurvenhälfte Entbehrliches angewandt, und hier wäre mit Fortbildung und Leitlinien anzusetzen. Zudem ergäben sich weitere Vorteile. Da Patienten mit Migräne von Hausärzten und Neurologen behandelt werden, würde ersichtlich, ob die Kurven deckungsgleich sind. Und da der vorbestehende Gesundheitszustand der Patienten bezeichnet wird, würden jene Ärztinnen, welche Chronischkranke nicht abschieben, oft ihre Anova-Auffälligkeit los. Zudem liesse sich feststellen, wo es zu den grossen Kostensteigerungen kommt, ob bei den Diabetikern oder in der Onkologie.

Funktioniert das Vorgehen, könnte man den TARMED sauber putzen und auf den Mist werfen; denn entscheidend ist nun, ob man die Mehrzahl der Patientinnen mit Migräne zu angemessenen Kosten behandelt. Vor allem aber würde ersichtlich, dass bei einzelnen Behandlungen die Kosten begründbar steigen und bei anderen gleich bleiben oder fallen, und die Kostenneutralität wäre kein Thema mehr.

\section{Bekämpfung des «Dällebach-Prinzips»}

(Man sucht den Fünfliber nicht dort, wo er verloren ging, sondern dort, wo man Licht hat): Jede schwergewichtig diagnostische Fachgesellschaft bezeichnet eine bis drei teure Untersuchungen, und bei diesen hat derjenige, welcher sie anordnete, bei Erhalt des Resultats anzugeben, ob dieses die Behandlung tatsächlich beeinflusst. Wie dargelegt [2], bräuchte es sieben typische Angaben; denn auch ein negativer Befund kann entscheidend sein (z.B. keine Metastasen bei nachgewiesenem Tumor), oder ein positiver Befund kann unerheblich sein (z.B. Altersveränderungen an der Wirbelsäule bei Rückenschmerzen).

\section{Numismatik}

Jede schwergewichtig therapeutische Fachgesellschaft bezeichnet ausgewählte Behandlungen, und bei diesen nennt die Patientin vor dem Beginn ihre Beschwerden und nach dem Abschluss der Behandlung den Erfolg [2]. (Stellt ein Patient kaum eine Besserung seines Sehvermögens fest, war die Kataraktoperation vermutlich locker indiziert.)

Die Vorgehen 2 und 3 werden durch die Fachgesellschaften überprüft, könnten jedoch freiwillig sein; wer mitmacht, darf sein Bemühen um effiziente Medizin sichtbar machen, zum Beispiel in Form einer Kennzeichnung neben dem "Arzt FMH».

\section{Ausblick}

Eine anfängliche Senkung, gefolgt von einem zwar unvermeidlichen, aber moderaten späteren Anstieg der Gesundheitskosten, würde nicht nur Goodwill schaffen, sondern es den Ärzten erlauben, ihre Anliegen besser durchzusetzen und gegenüber den Politikern den Spiess umzudrehen: Wir haben die Hausaufgaben gemacht, nun kümmert Euch um Eure Belange, wie monistische Finanzierung mit Mitgiften [2] und um die Preise zum Beispiel der Generika.

\section{Literatur}

1 Holzmann D, Straumann D. Indikation nach medizinischen oder ökonomischen Kriterien? Schweiz Ärztezeitung. 2016;97(6):218-9. Siehe dazu auch den Forumsbeitrag vom März 2016

2 Bracher D. Effizienzsteigerung im Gesundheitswesen. 2013. ISBN 978-3-033-04344-2.

\section{Bildnachweis}

(c) Ginasanders | Dreamstime.com 